\title{
An Overview on the Indium-Thallium (In-Tl) Shape Memory Alloy Nanowires
}

\author{
Zhiping P. Luo
}

Received: 3 October 2012/Accepted: 12 October 2012/Published online: 23 October 2012

(C) Springer Science+Business Media New York and ASM International 2012

\begin{abstract}
The binary indium-thallium (In-Tl) alloy is a classic type of shape memory alloy with a low melting temperature. In-Tl nanowires (NWs) were prepared by a simple mechanical injection of liquid metal method using anodized aluminum oxide (AAO) as a template. Sample preparation procedures are given in detail, including the AAO fabrication and mechanical injection techniques. Transmission electron microscopy (TEM) was utilized to characterize the NWs. It was revealed that the NWs were composed of polycrystals with high crystallinity. By in situ heating the NWs in the TEM, reversible transformations between the parent and the martensite phases were observed in both large and small NWs.
\end{abstract}

Keywords Electron microscopy - Indium-thallium (In-Tl) $\cdot$ Microstructure $\cdot$ Nanowire $\cdot$ Shape memory alloy

\section{Introduction}

Shape memory alloys (SMAs) have been widely studied in the field of metallurgy in the past, due to their wide range of practical applications [1]. Common classical examples of the SMA alloys include Ni-Ti [2], Ni-Al [3], Cu-Zn [4], $\mathrm{Cu}-\mathrm{Al}-\mathrm{Ni}$ [5], as well as the In-Tl system [6-10]; although, there are fewer studies on this alloy as compared with others. As early as 1972, Wayman and Shimizu [1] suggested the prerequisites for the shape memory behavior as that the (1) martensitic transformation is thermoelastic; (2) parent and martensitic phases are ordered; and (3) that

Z. P. Luo $(\bowtie)$

Department of Chemistry and Physics, Fayetteville State

University, Fayetteville, NC 28301, USA

e-mail: zluo@uncfsu.edu the martensite is internally twinned. In the In-Tl system with (15-30) at.\% $\mathrm{Tl}$, the crystal structure of the parent phase is face-centered cubic (fcc), while upon cooling these alloys undergo a martensitic transformation to a face-centered tetragonal (fct) structure with $c / a=1.03$, where $a$ and $c$ are the lattice parameters. The crystallographic relationship between these phases is shown in Fig. 1. In order to understand the shape memory behavior, the fcc-fct transformation and the twinning crystallography of the bulk samples were explored in detail $[6,7]$, as they are the key factors to link with their shape memory effect.

Nagasawa [9] demonstrated the shape memory effect of a narrow sheet sample with about $0.2 \mathrm{~mm}$ thickness in the composition of In-21 at.\% Tl. The sheet was bent into a ring shape at $130{ }^{\circ} \mathrm{C}$ when it was in the austenite state, and after it cooled down to room temperature (RT), it was deformed to flat. Upon heating back to $100{ }^{\circ} \mathrm{C}$, its shape turned to be curved, although not to a complete ring due to the limit of martensitic shears.

In recent years, nanostructures in metallic materials have attracted great attention because of the size effects on their properties [11-13]. In an early work, an open and fully connected Au ligament network structure was fabricated by electrochemical dissolution of $\mathrm{Ag}$ component from a miscible $\mathrm{Au}-\mathrm{Ag}$ alloy [11]. It was found that the yield strength of the $15 \mathrm{~nm}$ diameter ligaments was estimated to be $1.5 \mathrm{GPa}$, which was close to the theoretical strength of Au. Later, Dou and Derby [13] prepared isolated single gold nanowires (NWs), with wire diameters in the range $30-80 \mathrm{~nm}$, by electrodeposition into porous alumina templates and tested their mechanical properties. The maximum yield strength of the $30 \mathrm{~nm}$ diameter NWs was measured as approximately $1.4 \mathrm{GPa}$, which is significantly higher than polycrystalline gold specimens. To date, a number of metal NWs have been produced by 


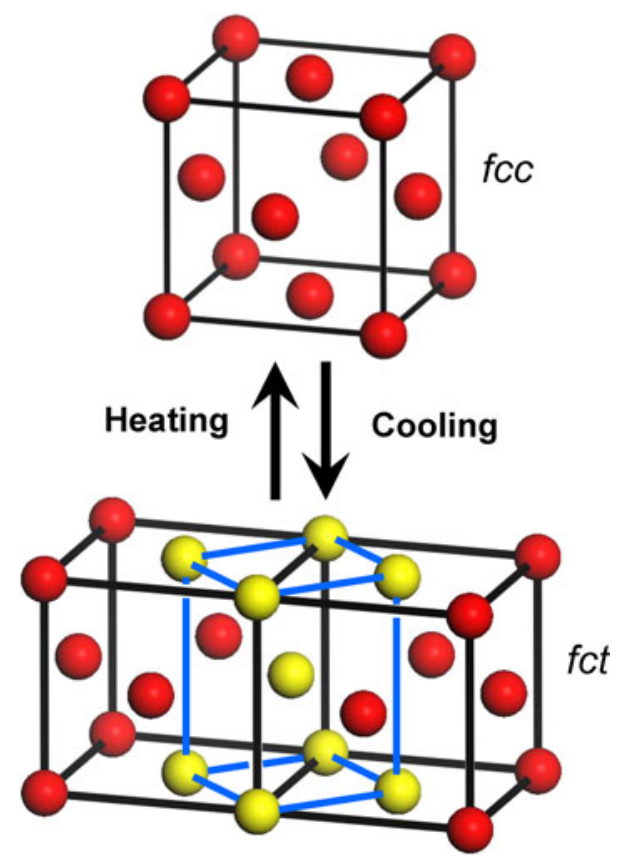

Fig. 1 Structure of the austenite (fcc) and martensite (fct) crystal phases

electrodeposition $[14,15]$ or mechanical injection of liquid metals [16-19].

In the In-Tl system, the alloy melting point is very low (lower than $304{ }^{\circ} \mathrm{C}$ for pure $\mathrm{Tl}$ ). Therefore, it is feasible to prepare the In-Tl NWs using the mechanical injection method. Recently, In-Tl NWs have been successfully fabricated $[20,21]$. This article presents an overview of the recent research on novel In-Tl-based SMA NWs.

\section{Experimental}

The experimental material was In-21 at.\% Tl, made by melting pure In and $\mathrm{Tl}$ in vacuum. In order to fabricate NWs, it is necessary to prepare the anodized aluminum oxide (AAO) template first. The schematic apparatus designed to prepare the AAO is shown in Fig. 2. A piece of aluminum plate with commercial purity $(\mathrm{Al}, 99.7 \%, 0.3 \mathrm{~mm})$ was connected to the anode side, while the counter electrode cathode material could be a piece of $\mathrm{Pt}$, carbon graphite, or even the same sample metal Al. Upon applying voltage to this circuit, $\mathrm{Al}$ dissolves into the acid solution which become $\mathrm{Al}^{3+}$, while $\mathrm{H}^{+}$ions were absorbed on the cathode side, which gain electrons to release $\mathrm{H}_{2}$ gas. Several major parameters, like voltage, the acid solution, temperature and time, all control the diameter and length of the yielded nanopores. To prepare small pores with diameters in the range of $10-25 \mathrm{~nm}, 15$ vol. $\% \mathrm{H}_{2} \mathrm{SO}_{4}$ acid was used at $19 \mathrm{~V}$; for medium pores in the range of $30-90 \mathrm{~nm}, 3 \mathrm{wt} . \% \mathrm{C}_{2} \mathrm{H}_{2} \mathrm{O}_{4}$ was used at $40 \mathrm{~V}$; and for large pores in the range of

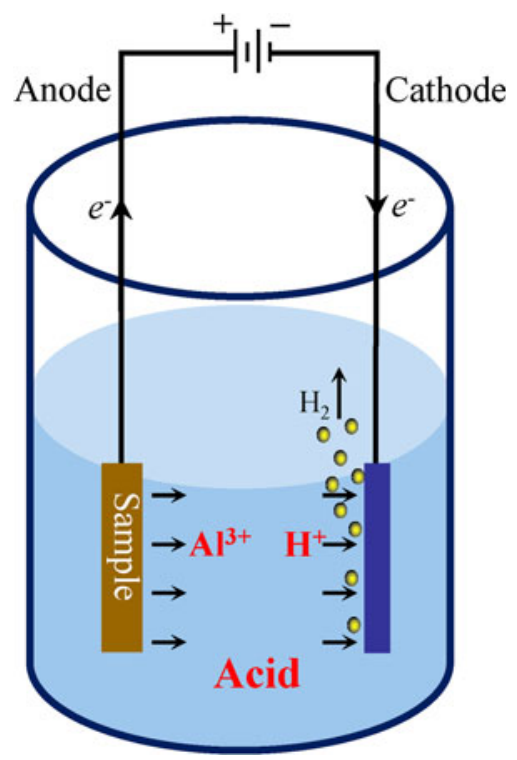

Fig. 2 Schematic of the apparatus to used prepare AAO template

$180-500 \mathrm{~nm}, 1$ wt. $\% \mathrm{H}_{3} \mathrm{PO}_{4}$ was used at $195 \mathrm{~V}$. Additional details about the preparation conditions are reviewed elsewhere [19]. Figure 3(a) shows a schematic structure of the as-prepared AAO. Highly ordered nanopores in a nearhexagonal pattern are formed on the top surface of $\mathrm{Al}$ foil. $\mathrm{A}$ barrier layer is formed on the walls of the AAO pores. Figure 3(b) and (c) are scanning electron microscope (SEM) images of two AAO samples, with pore size of 70 and $220 \mathrm{~nm}$ in diameter, respectively.

The In-Tl NWs were prepared by a mechanical injection method of liquid metal, as shown in Fig. 4. The AAO template was placed inside the piston vacuum chamber; a piece of $\mathrm{In}-\mathrm{Tl}$ metal was placed on top of the AAO template. The temperature of the bottom portion of the device was increased using an external heater. When the sample metal was completely molten, force was applied to the piston so that the melt could be injected into the AAO nanopores. The required pressure for the injection is discussed in Ref. [19]. On the upper side of the chamber near the rubber O-rings area, cooling water was supplied to protect the O-rings. The piston chamber is under vacuum to avoid the sample oxidation at high temperatures.

In order to release the NWs, the AAO template was dissolved in a basic $\mathrm{NaOH}$ solution. For the transmission electron microscopy (TEM) study, NWs were deposited on $\mathrm{Cu}$ grids that were coated with a pure carbon support film, and then observed at $200 \mathrm{kV}$; in situ heating was accomplished using a heating stage. The heating rate was approximately $10{ }^{\circ} \mathrm{C} / \mathrm{min}$, with a dwell time of approximately 5 min or longer to ensure the sample stability at each temperature. In order to minimize the electron beam damage, the areas to be viewed were moved away from the electron beam during the heating process. The pure carbon support film can withstand heating up to $600{ }^{\circ} \mathrm{C}$ without breaking. 
Fig. 3 a Schematic of the structure of AAO; b, c SEM images of as-fabricated AAO
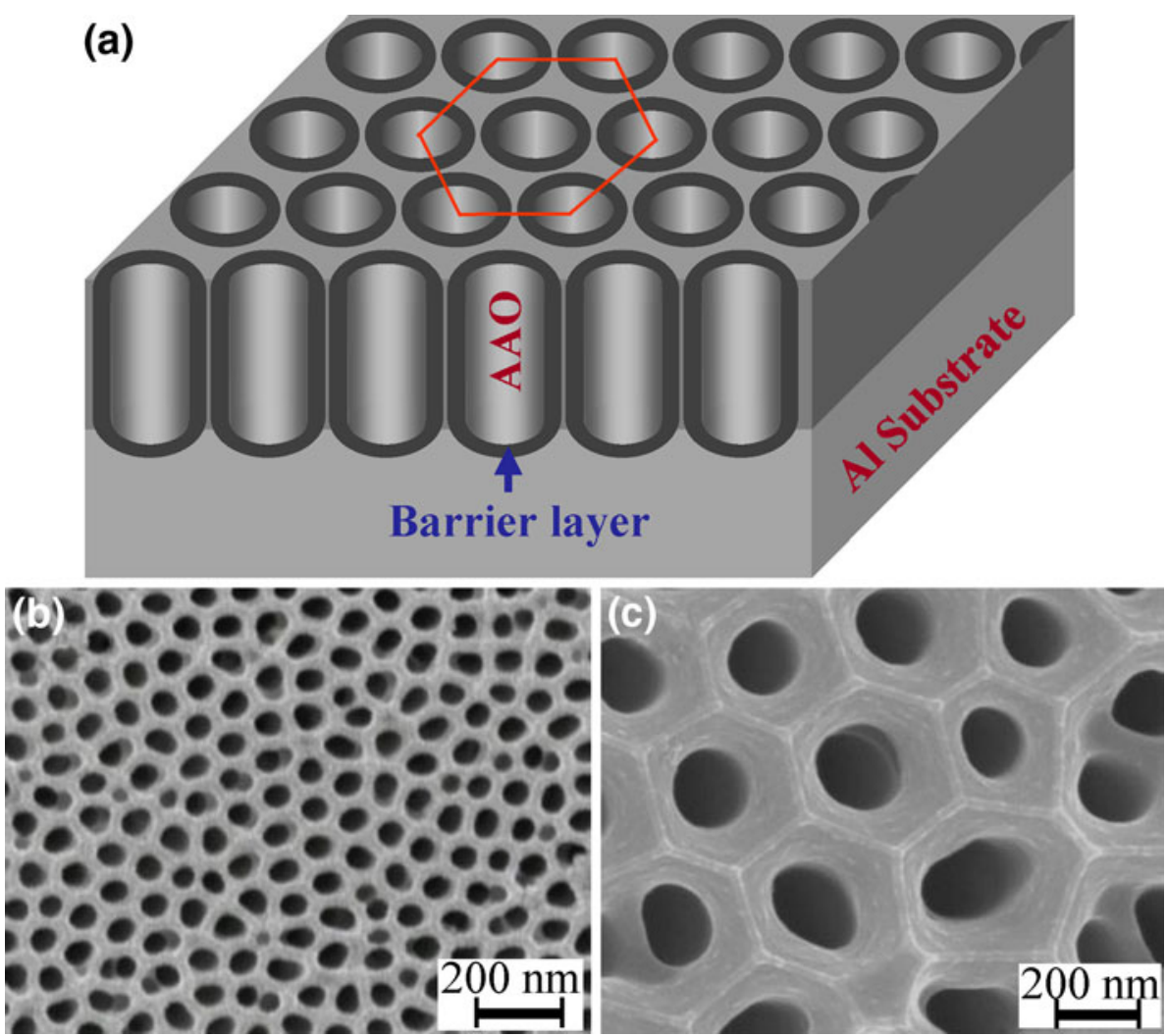

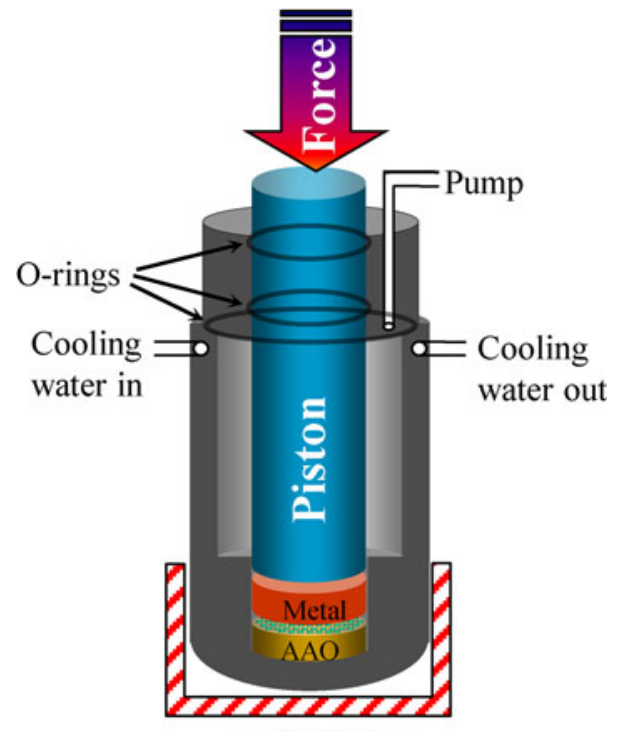

Heater

Fig. 4 Schematic of the apparatus used to prepare NWs by mechanical injection of liquid metal

\section{Results and Discussion}

X-ray diffraction analysis of the bulk experimental alloy was performed to identify the lattice parameters for this composition: $a_{\mathrm{fcc}}=0.47460 \mathrm{~nm}$ for the parent phase at a high temperature, and $a_{\mathrm{fct}}=0.47000 \mathrm{~nm}$ and $c_{\mathrm{fct}}=0.48343 \mathrm{~nm}$ $\left(c_{\mathrm{fct}} / a_{\mathrm{fct}}=1.029\right)$ for the fct phase at RT. As shown in Fig. 1, the Bravais lattice of the martensite fct phase is a body-centered tetragonal (bct, space group I4/ $\mathrm{mmm}$ ) structure, with $a_{\mathrm{bct}}=(\sqrt{2} / 2) a_{\mathrm{fct}}=0.33234 \mathrm{~nm} \quad$ and $\quad c_{\mathrm{bct}}=c_{\mathrm{fct}}=0.48$ $343 \mathrm{~nm}$. In this article, for simplification, the martensite structure is described as the fct structure to highlight its near fcc symmetry. The twinning plane in the In-Tl alloy is identified as $\{101\}$ type of the fct structure, which is consistent with the literature report on the pure indium sample [22].

In a previous study on pure indium nanoparticles (NPs), Oshima et al. [23] found that its structure is size dependent. When the NP size is above $5 \mathrm{~nm}$, the NPs exhibited a tetragonal structure that is similar to the bulk form; when the size is reduced below $5 \mathrm{~nm}$, the structure transformed to fcc. A similar fct-fcc transition was also found by Balamurugan et al. [24] in small indium NPs. However, in the case of 1D In-Tl NWs, the structure is found to be fct at $\mathrm{RT}$, and no size-induced phase transition is found ranging from 10 to $650 \mathrm{~nm}$ in diameter. The fact that the NWs retain the tetragonal martensitic structure in the NW is promising for future studies, as it is one of the prerequisites of the shape memory effect [1] on the nanoscale.

Figure 5 shows the selected-area diffraction (SAD) analysis of the prepared single NWs. In Fig. 5(a), there are two large NWs with diameters of $\sim 280$ and $220 \mathrm{~nm}$. The 

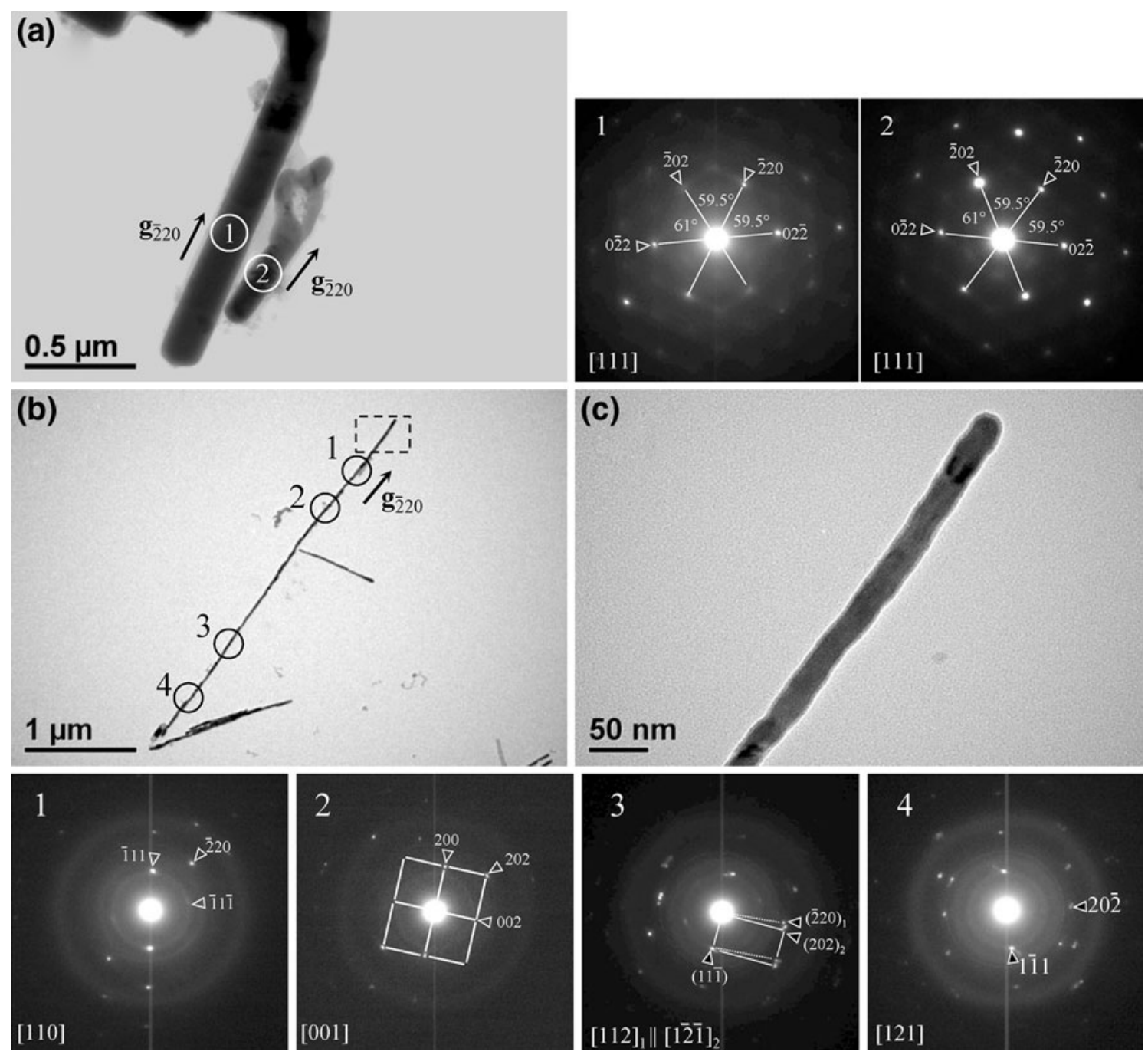

Fig. 5 SAD analysis of large NWs (a) and a small NW (b, c). The labeled SAD patterns are collected from the corresponding circled areas in the image

SAD patterns from the circled spots on these two NWs, labeled as 1 and 2, are shown on the right side of the image. Both of them exhibit the same geometry along [111] zone axes. Calculations show that the angle between $(0 \overline{2} 2)$ and $(\overline{2} 02)$ is $61^{\circ}$, while the angle between $(\overline{2} 20)$ and $(\overline{2} 02)$ and or between $(\overline{2} 20)$ and $(02 \overline{2})$ is $59.5^{\circ}$; therefore, the SAD patterns can be indexed according to the angle measurement, although their difference in lattice spacing is too small to measure from the SAD patterns. Note that $\mathbf{g}_{\overline{2} 20}$ is parallel to the wire axis, which means the wire growth direction is [110] rather than others like [011] or [101]. A smaller NW with a diameter of $25 \mathrm{~nm}$ is shown in Fig. 5(b), whose upper-right part as shown in the framed area is further magnified in Fig. 5(c). Four different spots along its length are chosen for the SAD analysis, as labeled as $1-4$, respectively. Spot 1 reveals a [110] zone axis pattern, with $\mathbf{g}_{220}$ near parallel to the wire axis, indicating that the wire growth direction is along [110]. However, the SAD pattern from spot 2 exhibits a [001] zone axis pattern, indicating that the NW is polycrystalline rather than a single crystal. Furthermore, spot 3 shows a compound pattern from two crystals, and spot 4 shows a pattern along [121] zone axis. All the NWs exhibit high crystallinity as shown from their SAD patterns.

Figure 6(a) shows NWs with a diameter of approximately $70 \mathrm{~nm}$. Also, note that these NWs are aggregated, which necessitated the use of a larger selective aperture, shown as the circle in the image. The SAD pattern for these wires at RT is shown in Fig. 6(b); the pattern contains polycrystalline rings from crystals along different orientations. However, a careful inspection reveals double rings; for example, (202) and (220) are indeed not in the same ring although their lattice spacings are very closing. This fact indicates that the structure is fct rather than fcc. When this area is heated to 
Fig. 6 In situ heating of aggregated NWs (70 nm in diameter). The SAD pattern is collected from the circled area in (a) at RT (b), which was heated up to $101{ }^{\circ} \mathrm{C}(\mathbf{c})$ and then cool down to RT (d)

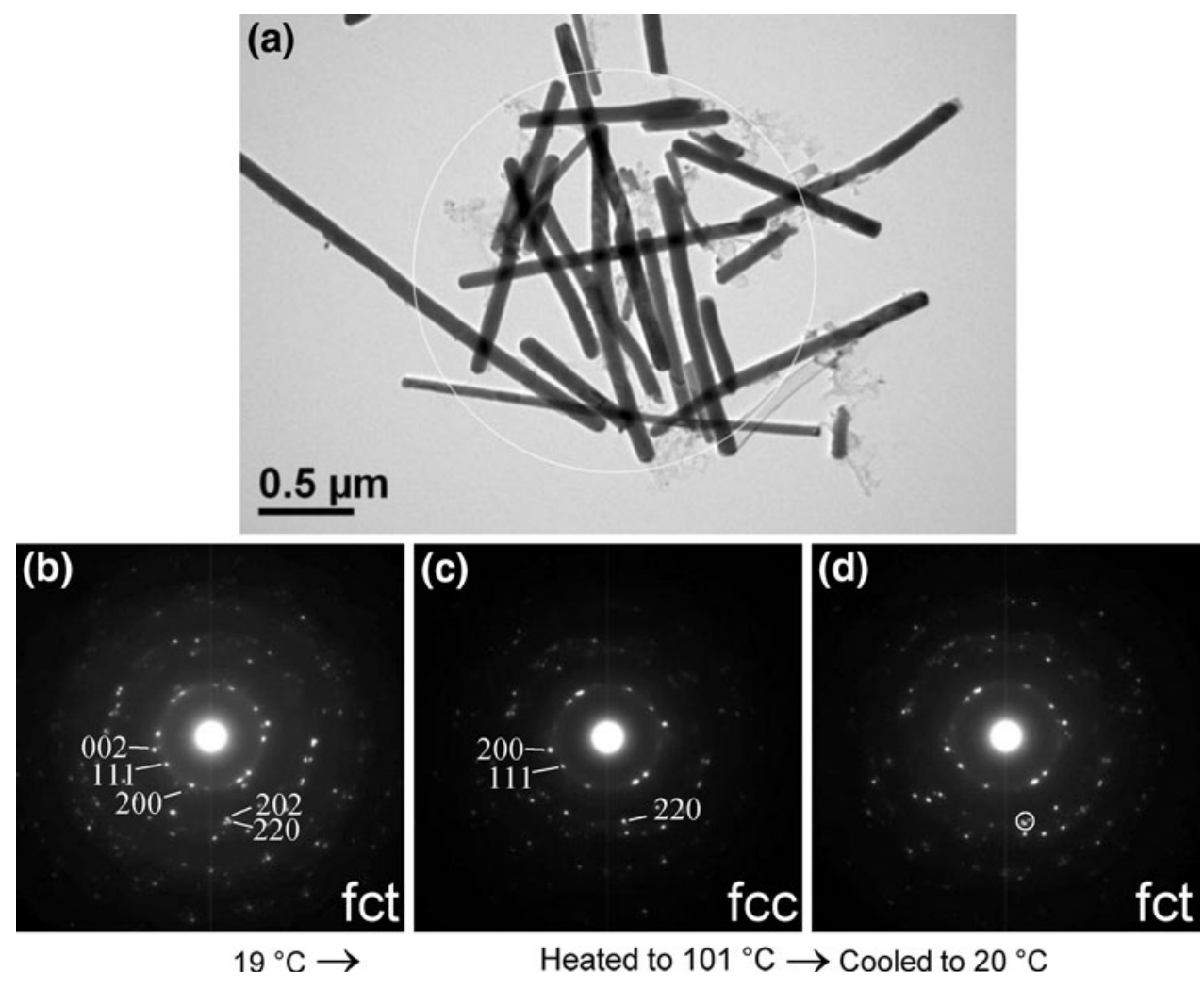

$101{ }^{\circ} \mathrm{C}$, the double rings are replaced with single rings, as shown in Fig. 6(c), which implies that the structure is changed to fcc at this temperature. However, when this area is cooled down to RT, double rings reappear as indicated in the circled spots in Fig. 6(d). Therefore, the fct-fcc transformation is found to be reversible.

An in situ heating experiment was also performed on the aggregated NWs with a diameter of approximately $15 \mathrm{~nm}$, as shown in Fig. 7. The low-magnification image in (a) indicates that some of these NWs have attached the $\mathrm{Cu}$ grid bar at the topside of the image, so that they can achieve better thermal conduction with a smaller temperature gradient than is normally observed for these experiments. Figure 7(b) is a magnified region of the circled area in (a), taken in the bright-field imaging mode. This area was selected for the in situ heating experiment, which is depicted at different temperatures in dark-field (DF) images in (c-f). The DF imaging mode highlights the internal nanocrystals along different orientations. At RT, as shown in Fig. 7(c), the DF image exhibits dense martensite internal twins. The SAD pattern from this area is shown in the inset, and depicts polycrystalline rings. The first ring is from (111), followed by double rings of (002) and (200), which is similar to Fig. 6. A quantitative processing of the SAD data using the procedures described in Ref. [25] clearly depicts double peaks [21]. Hence, the structure at RT is fct. However at $140{ }^{\circ} \mathrm{C}$, as shown in (d), the NWs exhibits almost uniform contrast without the contrast of twins, although some diffraction contrast is still visible due to the local orientation variations. From the associated SAD pattern, the location of the previous (002) and (200) double rings merges into a single (200) ring, indicating the fcc structure exists at this temperature. Due to the disappearance of twins in the NWs, the number of crystallites is significantly reduced, and thus fewer reflections appear in the pattern; the contribution from the amorphous carbon support film becomes more apparent. A similar image and associated SAD pattern are obtained at $160{ }^{\circ} \mathrm{C}$, as shown in (e). After cooling down to RT, the twins reappear in the NWs, as shown in (f). The SAD pattern indicates the fct martensitic structure, as shown in the inset in (f). This suggests that the fct-fcc transformation in the small NWs is also reversible.

\section{Conclusions}

By taking advantage of the low melting point of the In-Tl alloy, NWs were fabricated by the simple mechanical injection method of the liquid metal using AAO as templates. Detailed TEM studies indicated that the NWs are polycrystalline but with high crystallinity and defined crystallographic orientations. In either larger or small NWs, reversible transformations between the parent fcc and martensite fct were observed. 

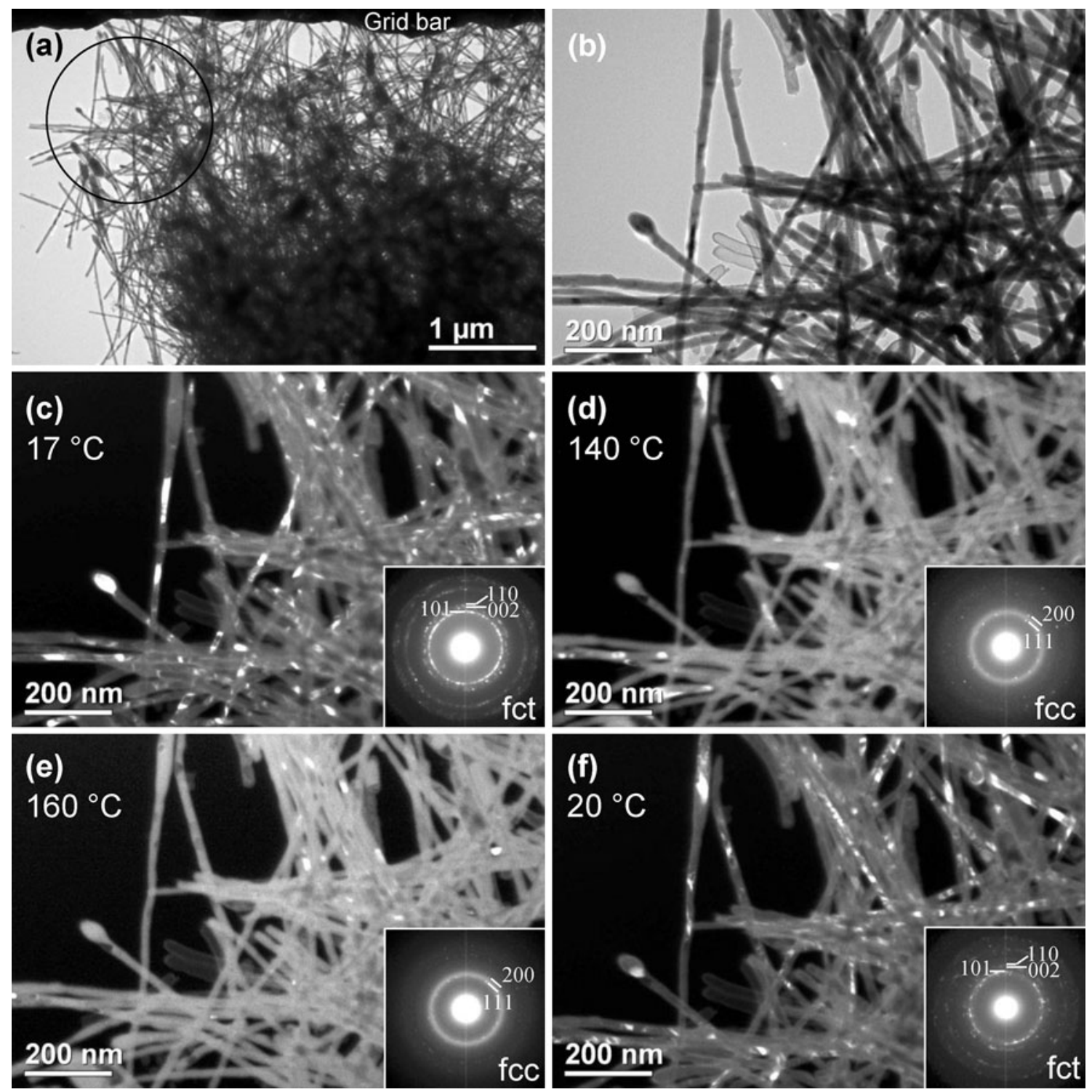

Fig. 7 In situ heating of aggregated small NWs (15 nm in diameter). a Low-magnification image; b magnified from (a), bright-field; $\mathbf{c}$ DF image at RT; d DF image after heating to $140{ }^{\circ} \mathrm{C}$; e DF image after heating to $160{ }^{\circ} \mathrm{C}$; $\mathbf{f}$ DF image after cooling down to $20^{\circ} \mathrm{C}$

Acknowledgments The experiment work was performed at Texas A\&M University. The author is grateful to his colleagues in references [16-21] for providing the samples and valuable discussions.

\section{References}

1. C.M. Wayman, K. Shimizu, The shape memory ('Marmem') effect in alloys. Met. Sci. J. 6(1), 175-183 (1972)

2. W.J. Buehler, J.V. Gilfrich, R.C. Wiley, Effect of low temperature phase changes on the mechanical properties of alloys near composition TiNi. J. Appl. Phys. 34(5), 1475-1477 (1963)

3. S. Rosen, J.A. Gobel, Crystal structure of nickel-rich NiAl and martensitic NiAl. Trans. Am. Inst. Min. Metall. Eng. 242(4), 722-724 (1968)
4. C.M. Wayman, On memory effects related to martensitic transformations and observations in $\beta$-brass and $\mathrm{Fe}_{3} \mathrm{Pt}$. Scripta Met. 5(6), 489-492 (1971)

5. K. Otsuka, K. Shimizu, Memory effect and thermoelastic martensite transformation in $\mathrm{Cu}-\mathrm{Al}-\mathrm{Ni}$ alloy. Scripta Met. 4(6), 469-472 (1970)

6. J.S. Bowles, C.S. Barrett, L. Guttman, Crystallography of cubictetragonal transformation the indium-thallium system. Trans. Am. Inst. Min. Metall. Eng. 188, 1478-1485 (1950)

7. Z.S. Basinski, J.W. Christian, Crystallography of deformation by twin boundary movements in indium-thallium alloys. Acta Met. 2(1), 101-116 (1954)

8. J.T.A. Pollock, H.W. King, Low temperature martensitic transformations in In/TI alloys. J. Mater. Sci. 3(4), 372-379 (1968)

9. A. Nagasawa, Memory effect in In-Tl alloy. J. Phys. Soc. Jpn. 30(4), 1200-1201 (1971) 
10. C.H. Sonu, T.J. O'Keefe, Characterization of phase-transformation behavior in electrolytically produced indium-thallium shapememory alloy-films. Mater. Charact. 33(4), 311-319 (1994)

11. C.A. Volkert, E.T. Lilleodden, D. Kramer, J. Weissmuller, Approaching the theoretical strength in nanoporous Au. Appl. Phys. Lett. 89(6), 89061920 (3 pp.) (2006)

12. D. Lee, X. Wei, X. Chen, M. Zhao, S.C. Jun, J. Hone, E.G. Herbert, W.C. Oliver, J.W. Kysara, Microfabrication and mechanical properties of nanoporous gold at the nanoscale. Scripta Mater. 56(5), 437-440 (2007)

13. R. Dou, B. Derby, The strength of gold nanowire forests. Scripta Mater. 59(2), 151-154 (2008)

14. Z.X. Ye, H. Zhang, H.D. Liu, W.H. Wu, Z.P. Luo, Observation of superconductivity in single crystalline Bi nanowires. Nanotechnology 19(8), 085709 (5 pp.) (2008)

15. Z.X. Ye, H.D. Liu, Z.P. Luo, H.G. Lee, W.H. Wu, D.G. Naugle, I. Lyuksyutov, Thickness dependence of the microstructures and magnetic properties of electroplated Co nanowires. Nanotechnology 20(4), 045704 (8 pp.) (2009)

16. C.C. Chen, Y. Bisrat, Z.P. Luo, R.E. Schaak, C.G. Chao, D.C. Lagoudas, Fabrication of single-crystal tin nanowires by hydraulic pressure injection. Nanotechnology 17(2), 367-374 (2006)

17. Y. Bisrat, Z.P. Luo, D. Davis, D. Lagoudas, Highly ordered uniform single-crystal $\mathrm{Bi}$ nanowires: fabrication and characterization. Nanotechnology 18(39), 395601 (6 pp.) (2007)

18. S.H. Chen, C.C. Chen, Z.P. Luo, C.G. Chao, Fabrication and characterization of eutectic bismuth-tin (Bi-Sn) nanowires. Mater. Lett. 63(13-14), 1165-1168 (2009)
19. C.C. Chen, D. Fang, Z.P. Luo, Fabrication and characterization of highly-ordered valve-metal oxide nanotubes and their derivative nanostructures: a review. Rev. Nanosci. Nanotechnol. 1(4), 28 pp. (2012). doi:10.1166/rnn.2012

20. F.R. Phillips, D. Fang, H.X. Zheng, D.C. Lagoudas, Phase transformation in free-standing SMA nanowires. Acta Mater. 59(5), 1871-1880 (2011)

21. H.X. Zheng, Z.P. Luo, D. Fang, F.R. Phillips, D.C. Lagoudas, Reversible phase transformations in a shape memory alloy In-Tl nanowires observed by in situ transmission electron microscopy. Mater. Lett. 70(1), 109-112 (2012)

22. G. Remaut, A. Lagasse, S. Amelinckx, The electron microscopic observation of mechanical twins in indium. Phys. Status Solidi 6(3), 723-731 (1964)

23. Y. Oshima, T. Nangou, H. Hirayama, K. Takayanagi, Face centered cubic indium nano-particles studied by UHV-transmission electron microscopy. Surf. Sci. 476(1-2), 107-114 (2001)

24. B. Balamurugan, F.E. Kruis, S.M. Shivaprasad, O. Dmitrieva, H. Zähres, Size-induced stability and structural transition in monodispersed indium nanoparticles. Appl. Phys. Lett. 86(8), 083102 (3 pp.) (2005)

25. Z.P. Luo, Y. Vasquez, J.F. Bondi, R.E. Schaak, Pawley and Rietveld refinements using electron diffraction from $L 1_{2}$-type intermetallic $\mathrm{Au}_{3} \mathrm{Fe}_{1-x}$ nanocrystals during their in situ orderdisorder transition. Ultramicroscopy 111(8), 1295-1304 (2011) 